\title{
High-Precision Indoor Flight path Tracking using UWB Sensor in a Thermal Power Plant Boiler
}

\author{
In-Kyum Kim, Won-Hyuck Choi and Min-Seok Jie* \\ Dept. of Aeronautical System Engineering, Hanseo University, Taean, \\ Republic of Korea \\ Kik6040@naver.com,choiwh@hanseo.ac.kr,jiems@hanseo.ac.kr
}

\begin{abstract}
The use of Unmanned Arial Vehicle(UAV) began with military reconnaissance, and has recently utilized environmental monitoring, traffic monitoring, and entertainment markets.

In this unmanned aerial vehicle, posture control based on Micro Electro Mechanical System(MEMS) sensor and flight position correction using Global Positioning System(GPS) are performed. MEMS that controls attitude of UAV is composed of multiple sensors to minimize errors.

The GPS receives the position information of the UAV through four or more satellites to know the flight position. It is possible to accurately locate the unmanned airplane or carry out mission flight to the destination.

MEMS and GPS are essentially used to perform missions on unmanned aerial vehicles. Unmanned aerial vehicles for military reconnaissance use GPS to know the exact location on the opposite side of the globe, and carry out autonomous flight and automatic landing with high accuracy.

In the non-GPS area where the GPS signal is not received, it is impossible to know the position of the flight position and the destination of the unmanned airplane, and it is impossible to perform the mission such as autonomous flight and automatic landing.

In the non-GPS indoor space, a sensor is required to replace GPS. In case of unmanned aircraft, position estimation using signal strength of Wi-fi and Bluetooth communication in a narrow space, hence accuracy and performance degradation due to space and obstacles occur.

In this paper, the accuracy of IPS (Indoor Positioning System) using UWB (Ultra Wide Band) communication was proposed by comparing the accuracy of UWB tracking of unmanned airplanes using $U W B$.

Also, UWB IPS performance was verified through automatic hovering mission flight and automatic landing test for flight path tracking and accuracy verification experiments of UAVs using UWB communication in the indoor space of boiler in non-GPS condition of special industrial field.
\end{abstract}

Keywords: thermoelectric power plant, indoor flight, precision flight, indoor positioning system, unmanned aerial vehicle, ultra wide band

\section{Introduction}

The use of unmanned aerial vehicles is rapidly being applied to industrial applications along with the 4th industry in military aircraft for military purposes. The traffic monitoring and environmental monitoring fields have achieved rapid diffusion and time efficiency by applying unmanned aerial technology to the commercialization stage.

Received (June 6, 2018), Review Result (August 4, 2018), Accepted (August 10, 2018)

* Corresponding Author 
UAV using LTE communication and GPS can fly long distances and return to the mission, and unlike human-controlled aircraft, they have been able to maximize the effectiveness of the mission.

UAV that performs missions outdoors can receive very accurate location information because they use GPS. In order to apply to industrial sites with special environments such as thermal power plants, communications should be performed to transmit position information such as MEMS sensors and GPS, which control the attitude of the UAV, and the received position data must be very precise.

The coal-fired thermal power plant has a boiler and the boiler performs periodic inspections. Figure 1 shows that the inside of the boiler was made of steel tube, and the operation of the power plant is being stopped during the inspection period.

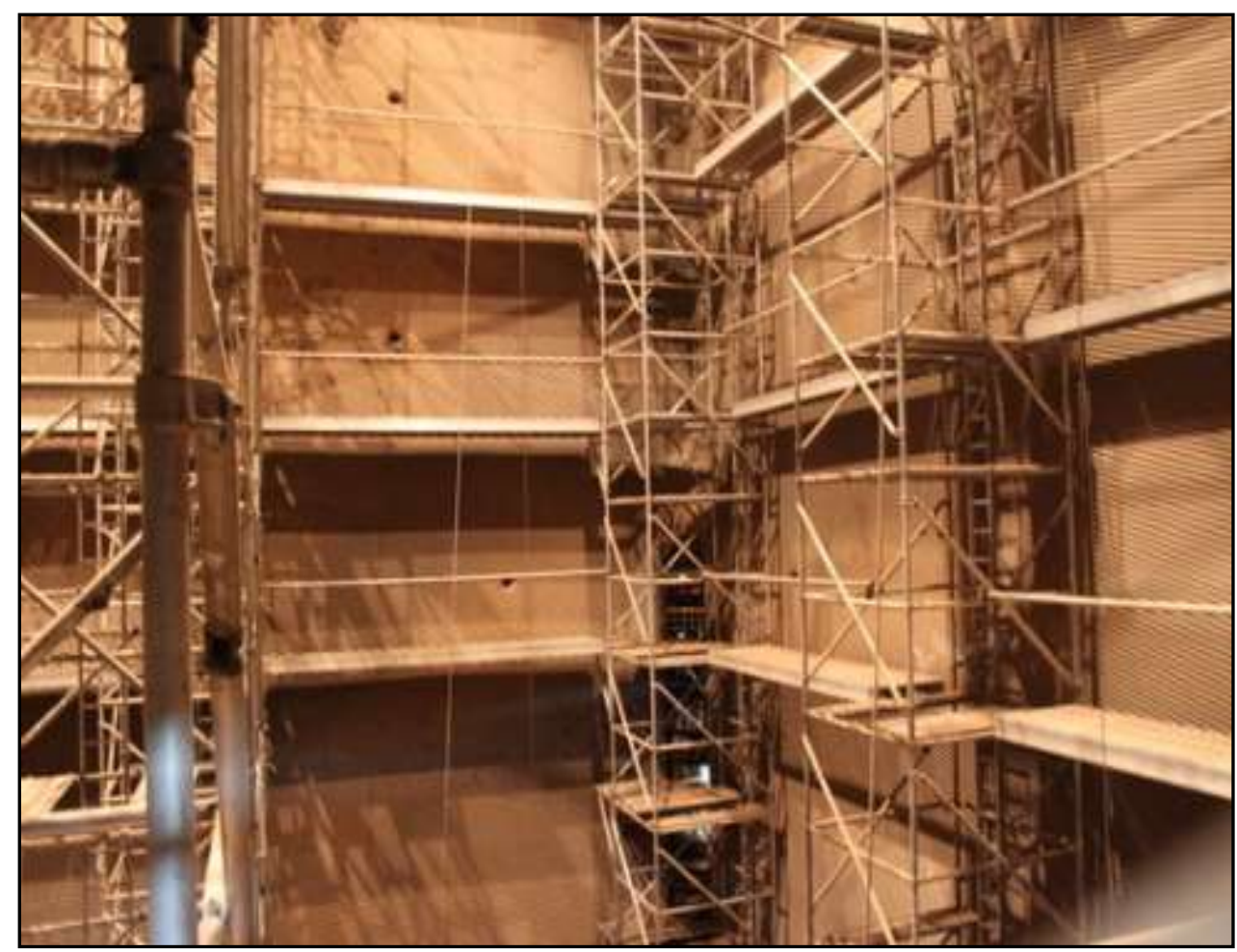

Figure 1. Power Plant Boiler

The duration of the boiler inspection is from 1 month to 6 months. Boiler inspection is carried out by visual inspection after about 15 operators had installed scaffolding. A safety accident such as a fall or injury could occur during the inspection.

Recently, unmanned aerial vehicle technology has been extended from outdoor missions to indoor flight mission, and studies are being conducted to estimate the indoor location using Wifi, Bluetooth, and Beacon communication strengths to estimate the location of unmanned aircraft.

The Indoor Positioning System using Wifi, Bluetooth and Beacon communication has a disadvantage of difficulty in estimating the accurate position when the signal strength becomes weak due to obstacles and walls.

Therefore, indoor flight using Wifi, Bluetooth, and Beacon communication can estimate the position in a small space, but the accuracy degrades in a large indoor space.

In this paper, IPS using Ultra Wide Band was applied to UAVs and compared the hovering flight of UAVs using GPS, UWB hovering and Waypoint flight position accuracy. 


\section{UWB Indoor Positioning System}

\section{1. 'UWB System}

The UWB Anchor and Tag used in this study were based on the Poxyz development kit with Decawave's DW1000. Figure 2 shows an anchor and tag of UWB system. Tag is composed of Arduino shield in combination with Arduino Uno process to receive UWB position information.

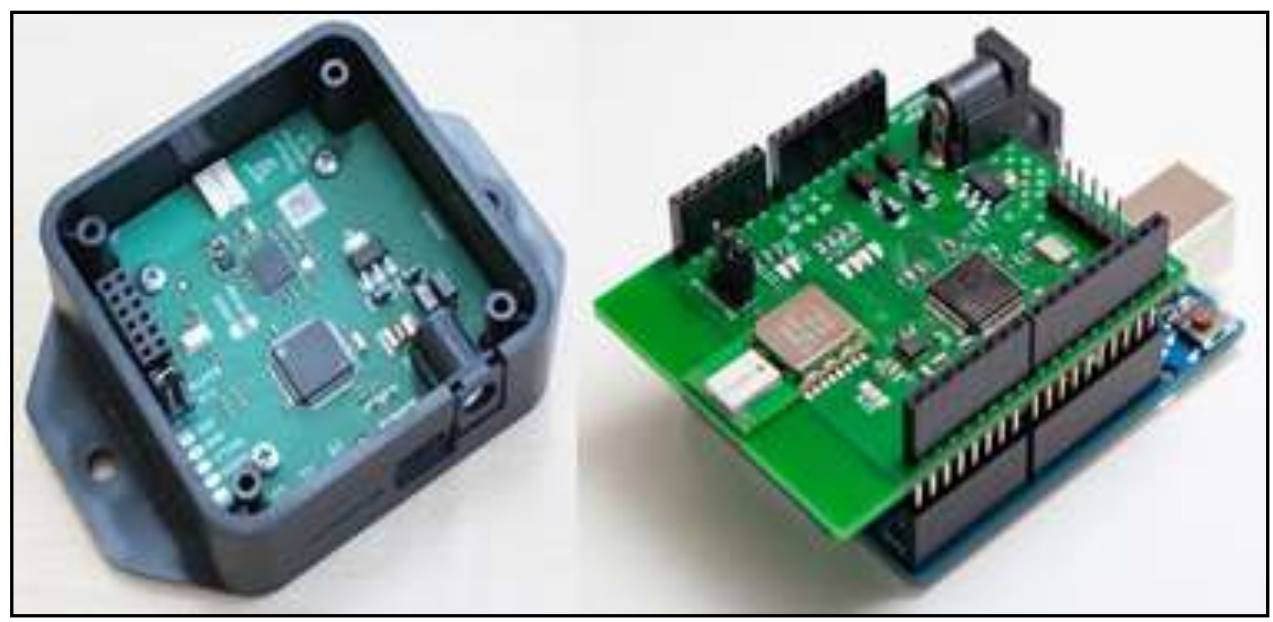

Figure 2. UWB System Anchor and Tag with Arduino(Uno)

In case of communication using existing Wifi, Bluetooth, and Beacon, there is an error in position accuracy due to multipath.

In the case of the IR-UWB system (Impulse-Radio Ultra-Wide Band), since the multi-path resolution with respect to time was precisely used because of the characteristic of the ultra-short pulse, Imaging communication, and the likes. UWB communication can be defined as a system satisfying different conditions.

$$
\begin{aligned}
& \text { Center Grequency }=\frac{f_{H}+f_{L}}{2} \\
& \text { Fractional Bandwidth }=\frac{f_{H}-f_{L}}{\left(f_{H}+f_{L}\right) / 2}
\end{aligned}
$$

First definition is in the Equation (1 2), and represent the upper and lower frequencies, respectively, with at the highest point of the signal spectrum.

The second definition is defined in Equation (2), a system with a bandwidth of $500 \mathrm{MHz}$ or more, regardless of the fractional bandwidth.

\section{2. 'UWB Signal}

Due to the nature of the signals, UWB pulses can be distinguished even in noisy environments, plus the signals are resistant to multipath effects. All of these traits give UWB big advantage over traditional narrowband signals in case of ranging capabilities. Also due to the strict spectral mask, the transmitting power lies at the noise floor, which means that UWB does not interfere with other radio communication systems operating in the same frequency bands, since it just increases the overall noise floor, a principle that is very similar to spread spectrum technologies (CDMA). 


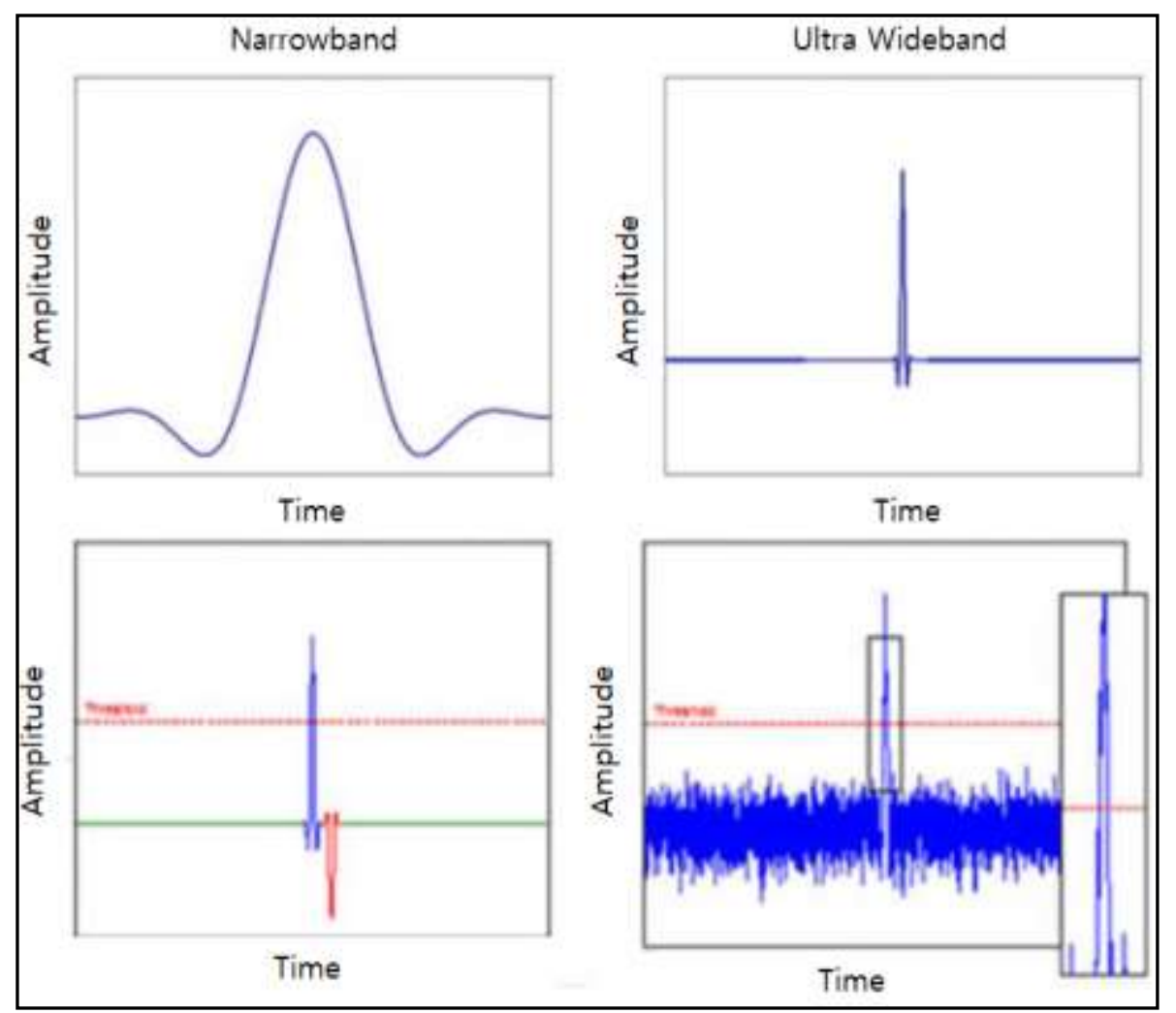

Figure 3. UWB Signal Spectrum

\subsection{2-Dimensional Position Location}

The triangulation method is a method of using the geometric calculation of the real-time position of a moving object in a plane in a general RTLS (Real-Time Location System). More than three APs were required to track the target. In Figure 4, the distances of the three APs can be obtained from Equation $(3 \sim 5)$, where d1, d2, and $\mathrm{d} 3$ are the distance to each tag.

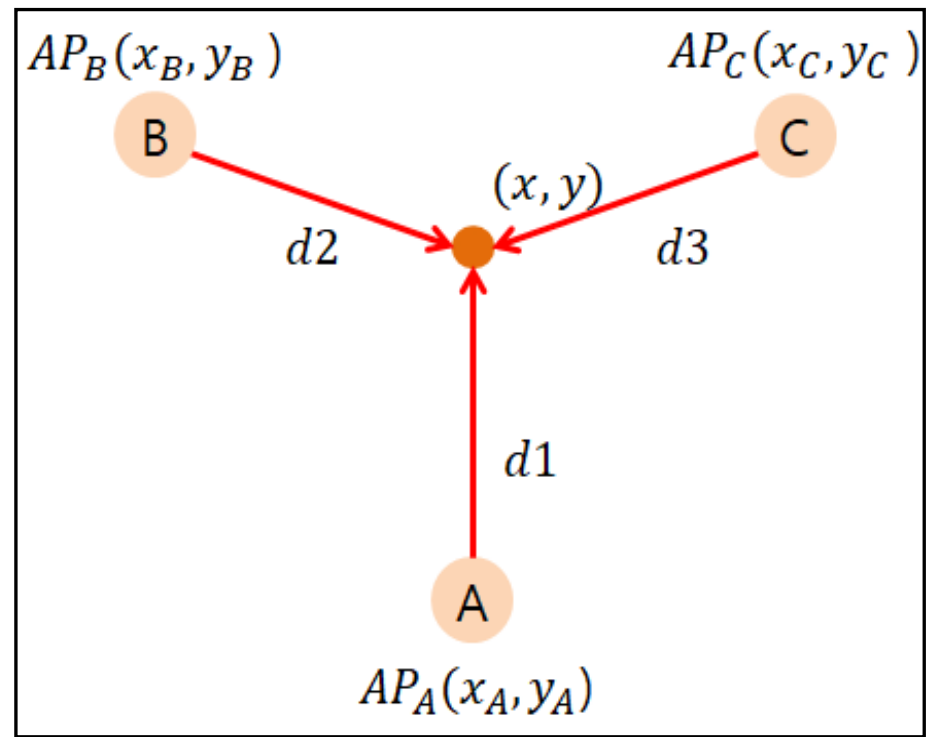

Figure 4. Two-dimensional Position Prediction 


$$
\begin{aligned}
& \left(x-x_{A}\right)^{2}+\left(y-y_{A}\right)^{2}=d 1^{2} \\
& \left(x-x_{B}\right)^{2}+\left(y-y_{B}\right)^{2}=d 2^{2} \\
& \left(x-x_{C}\right)^{2}+\left(y-y_{C}\right)^{2}=d 2^{2}
\end{aligned}
$$

The distance from each AP to the target point for position calculation can be calculated using Pythagorean Theorem. Two-dimensional coordinates can be calculated using Equation $(6 \sim 7)$.

$$
\begin{aligned}
& x=\frac{d 1^{2}+d 2^{2}+x_{A}^{2}}{2_{x A}} \\
& y=\frac{d 1^{2}+d 2^{2}+y_{A}^{2}}{2_{y A}}
\end{aligned}
$$

\subsection{UWB connection to Flight Computer}

The unmanned aircraft used in the test was a F450 Quadcopter, a Pixhawk Flight Computer using Ardupilot-based firmware, and UWB tags were connected to a serial port as shown in Figure 5 and data was transmitted at 115200 baud rate.

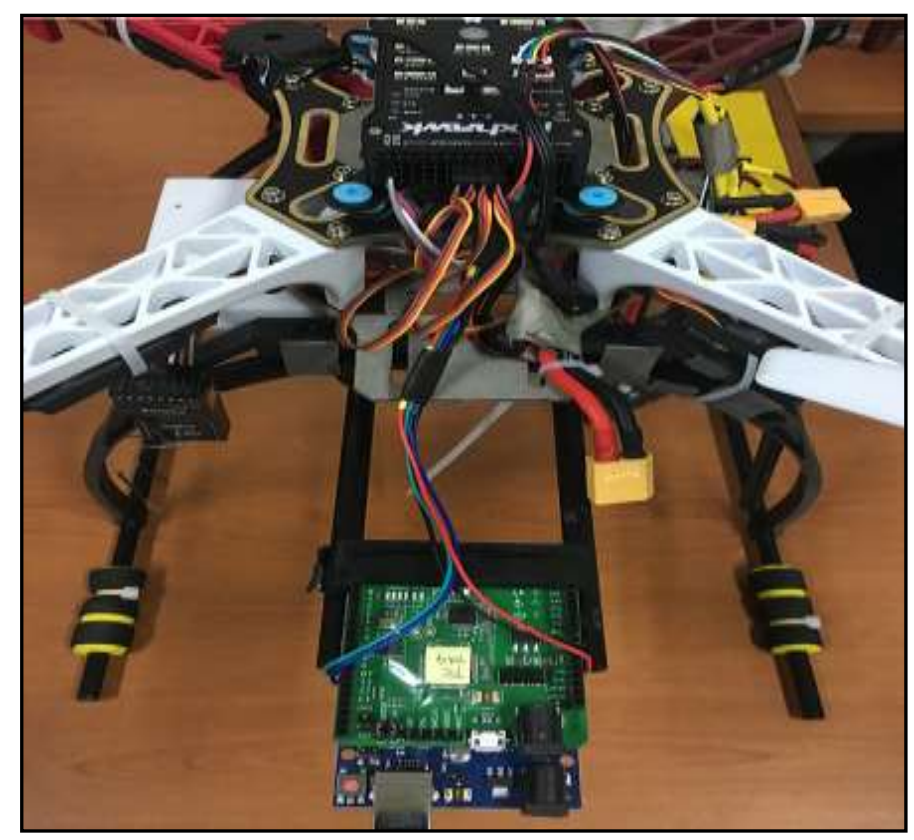

Figure 5. UWB Tag Connect to FC

\subsection{UWB, GPS Outdoor 2- Dimensional Experiment}

Figure 6 and Figure 7 track Waypoint flight of unmanned aerial vehicle using GPS in outdoor environment and Waypoint flight path of UAV using UWB. The distance between the waypoints is about $4 \mathrm{~m}$, and using GPS and UWB to confirm the arrival to the destination point. 


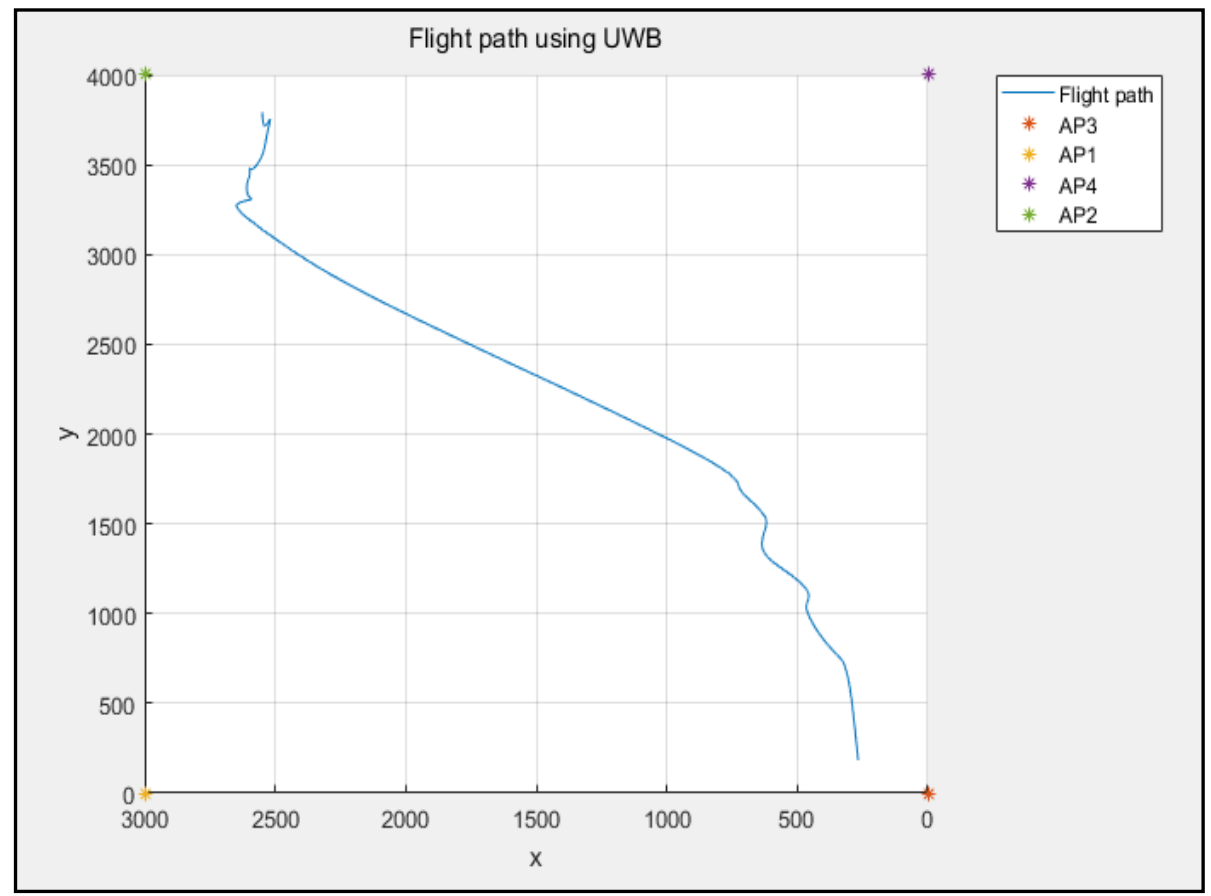

Figure 6. Outdoor 2D Tracking Using UWB

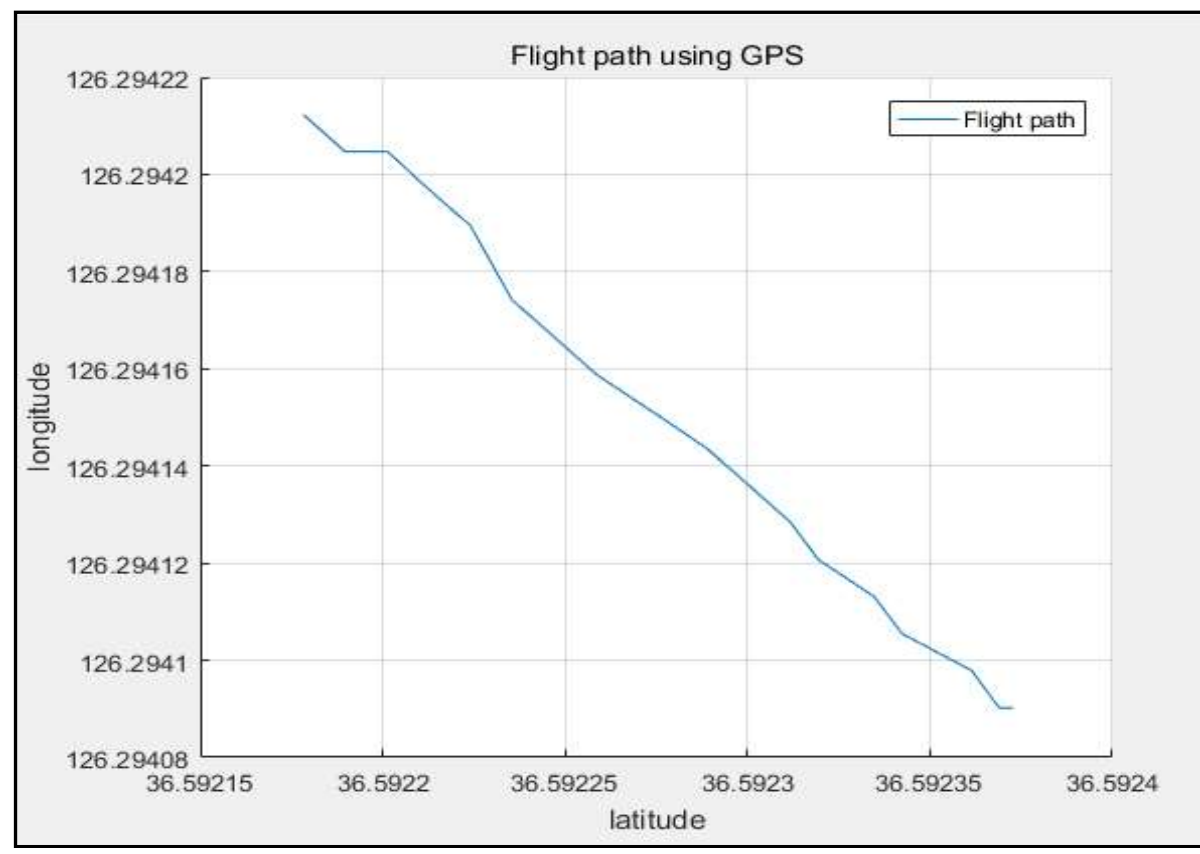

Figure 7. Outside 2D Tracking Using GPS

\subsection{3-Dimensional Position Location}

Three-dimensional positioning is shown in Figure 8. Four APs are required as shown in Figure 8. Assuming that each AP is at a distance of $L$, the formula of $x, y$, $\mathrm{z}$ can be obtained by using Equation (9). 


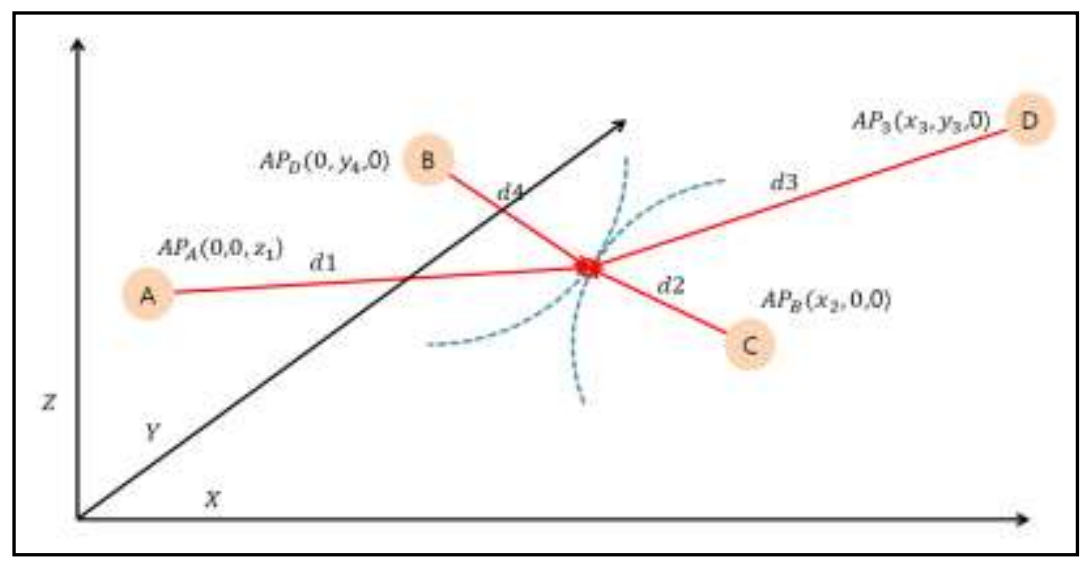

Figure 8. Indoor Positioning System

$\mathrm{z}=z_{1} \pm \sqrt{L^{2}-\left(x^{2}+y^{2}+z_{1}^{2}-L^{2}\right.}$

\subsection{UWB Indoor 3- Dimensional Experiment}

Figure 9 shows the results of the UWB-based positioning of the unmanned aircraft in an indoor test bed with a structure similar to that of a thermal power plant boiler. The test bed was $5 \mathrm{~m}$ in length, $5 \mathrm{~m}$ in height, and $7 \mathrm{~m}$ in height.

Four UWB anchors were placed to receive three-dimensional position data. 50 and 200 samples of position data of hovering flight were collected.

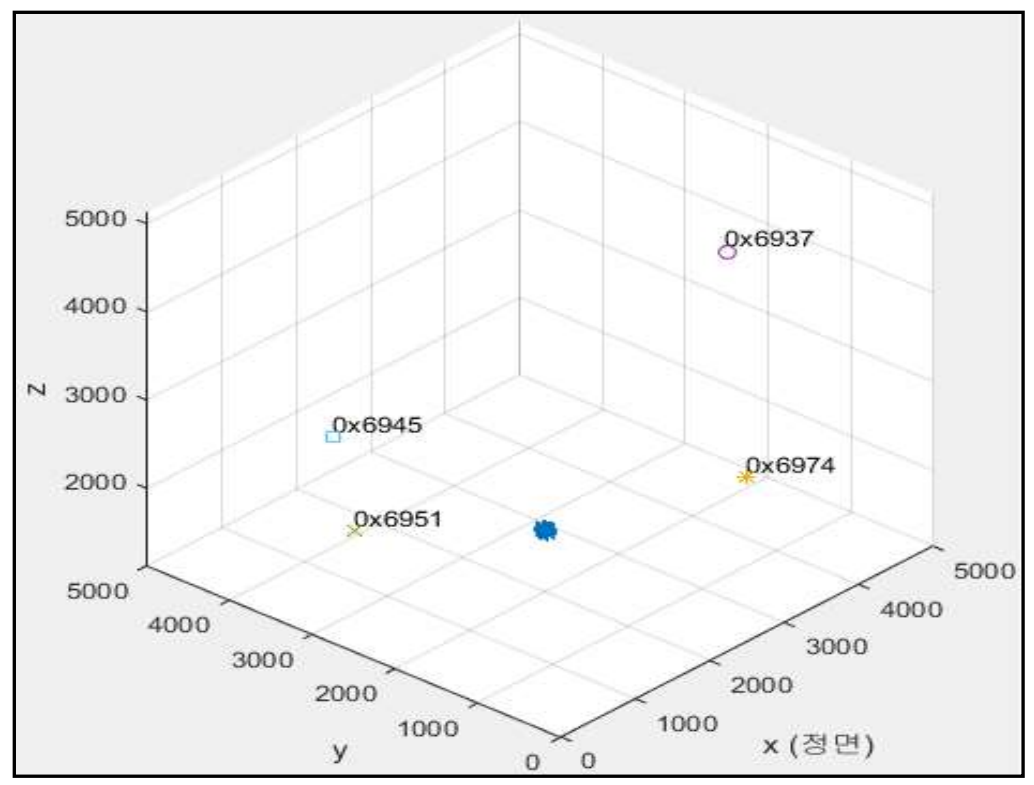

Figure 9. UAV Hovering Position Tracking

The hovering flight position data variance and standard deviation values for Fig. 9 are shown in Tabs 1 and 2 below.

Table 1. Hovering Flight Position Variation

\begin{tabular}{|c|c|c|r|}
\hline & X var & Y var & \multicolumn{1}{|c|}{ Z var } \\
\hline Sample 50 & $62.59 \mathrm{~mm}$ & $36.90 \mathrm{~mm}$ & $77.56 \mathrm{~mm}$ \\
\hline Sample 200 & $7.911 \mathrm{~mm}$ & $6.074 \mathrm{~mm}$ & $8.80 \mathrm{~mm}$ \\
\hline
\end{tabular}




\section{Table 2. Move Flight Position Standard Deviation}

\begin{tabular}{|c|c|c|c|}
\hline & \multicolumn{1}{|c|}{ X var } & \multicolumn{1}{|c|}{ Y var } & Z var \\
\hline Sample 50 & $80.25 \mathrm{~mm}$ & $82.85 \mathrm{~mm}$ & $124.67 \mathrm{~mm}$ \\
\hline Sample 200 & $8.95 \mathrm{~mm}$ & $9.10 \mathrm{~mm}$ & $11.165 \mathrm{~mm}$ \\
\hline
\end{tabular}

Figure $10-11$ show the results of tracking the flight position of UAV with UWB IPS in an indoor test bed space of $5 \mathrm{~m}, 5 \mathrm{~m}$, and $7 \mathrm{~m}$ height.

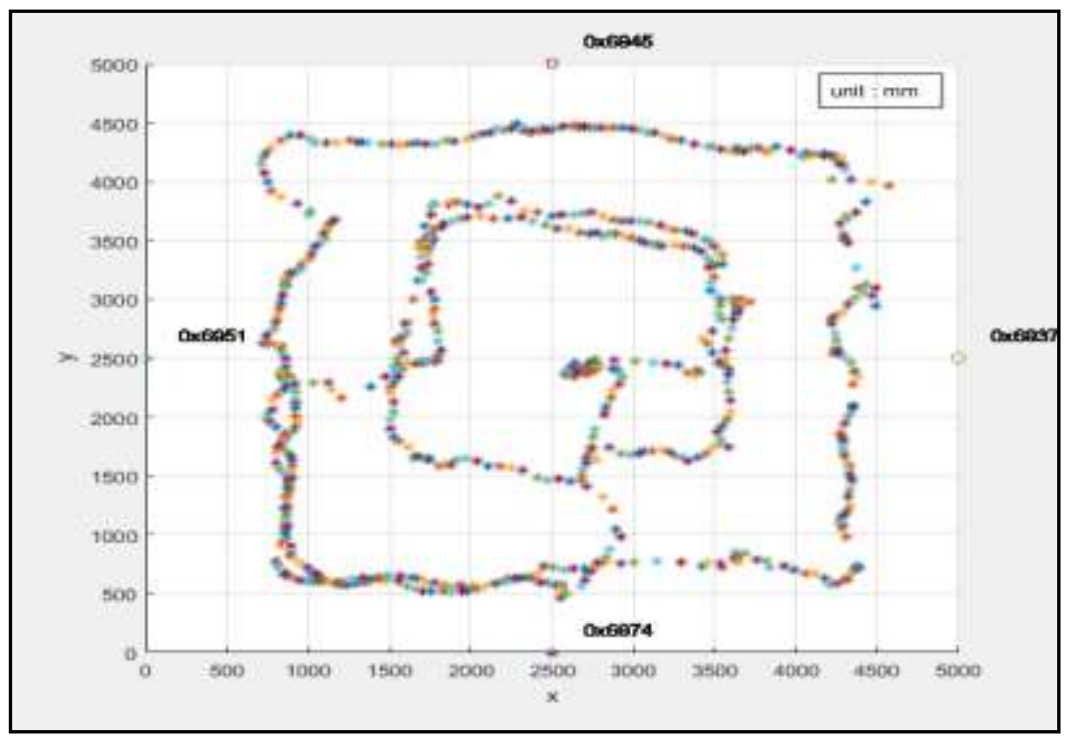

Figure 10. 3D Flight Paths Tracking (x, y)

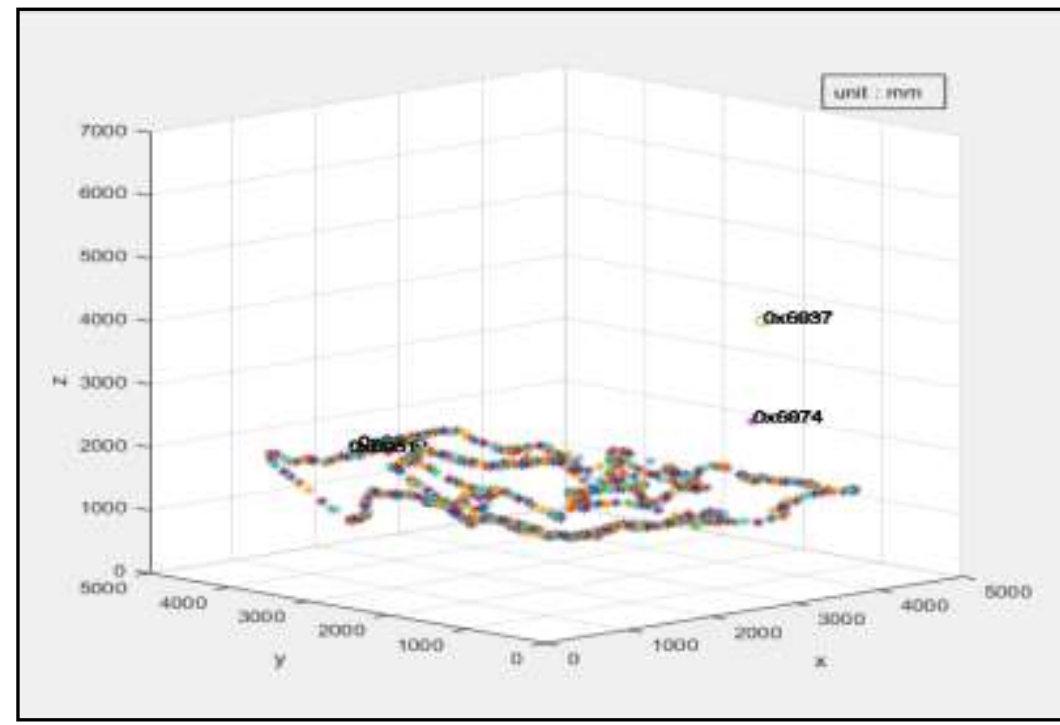

Figure 11. 3D Flight Paths Tracking $(x, y, z)$

Figure 12 - 13 show the results of tracking the flight position of UAV with UWB IPS in an indoor test bed space of $5 \mathrm{~m}, 5 \mathrm{~m}$, and $7 \mathrm{~m}$ height. 


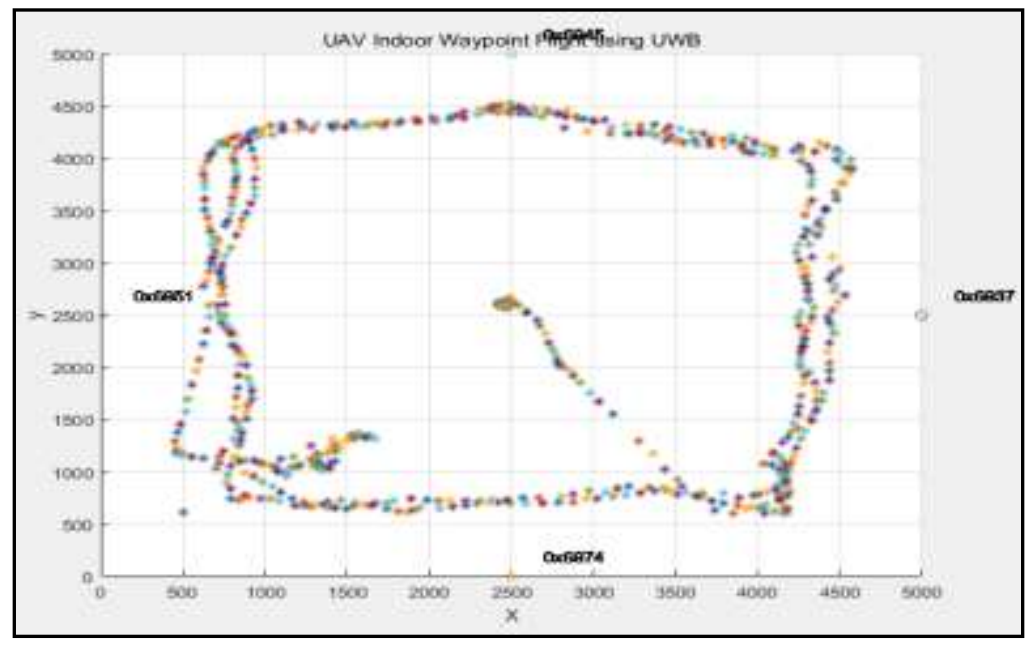

Figure 12. UAV Indoor Waypoint Flight using UWB(x, y)

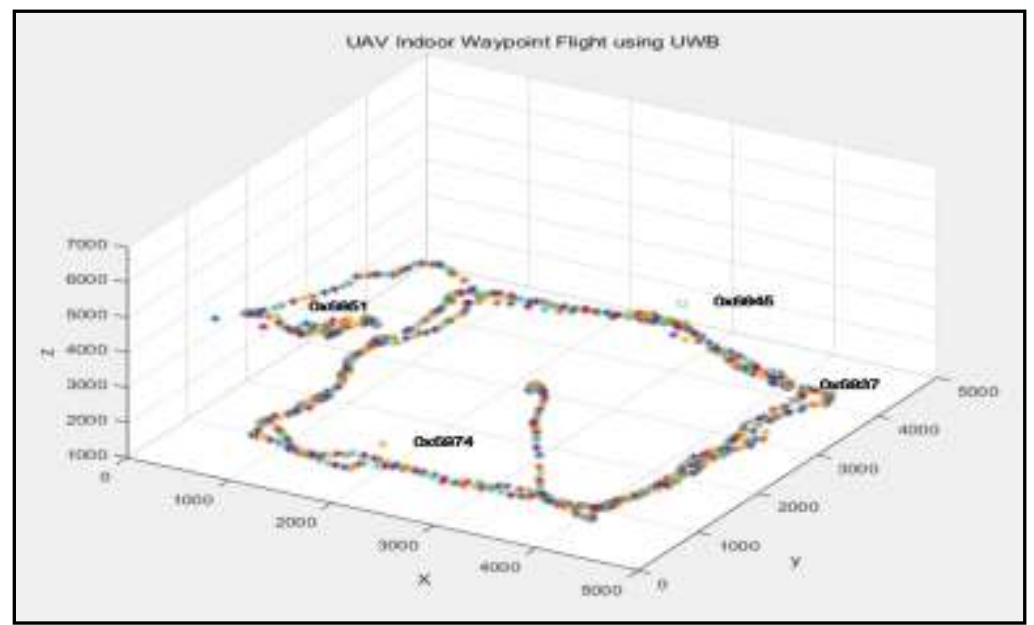

Figure 13. UAV Indoor Waypoint Flight using UWB(x, y, z)

\section{Conclusion}

Unmanned aeronautical technology is a technology that can be applied to various fields. In the meantime, it was being used together with GPS to receive an accurate position and perform a flight mission. In recent years, unmanned aerial technology has been spreading in compact and intensive form, and conditions for indoor flight and application of unmanned aerial vehicles in industrial fields are rapidly expanding.

In this paper, the way to reach the destination precisely was experimented on in case of unmanned aircraft using GPS and UWB in the field. In the indoor space of non-GPS state, UWB IPS was used to track indoor flight of UAV.

Four or more anchors were needed to carry out UWB communications. UWB IPS was used to verify the indoor flight of unmanned aircraft.

\section{Acknowledgments}

This paper is a revised and expanded version of a paper entitled [Indoor flight path tracking in thermal power plant boiler by using UWB positioning sensor] presented at $\left[10^{\text {th }}\right.$ International Conference on $\mathrm{u}$ - and e- Service, Science and Technology (UNESST2018), Hilton Hanoi Opera, Hanoi, Vietnam, July 12-14].

This work was supported by a grant from Hanseo University in 2018. 
This study was supported by the "Development of the boiler inspection system basedon UAV technology" research project of Korea Western Power co., Ltd.

This work was supported by the Korea Institute for Advencement of Technology(KIAT) grant funded by the Korean government(Motie : Ministry of Trade, Industry \& Energy) (No. N0002431).

\section{References}

[1] H. S. Nalwa, Editor, "Magnetic Nanostructures", American Scientific Publishers, Los Angeles, (2003).

[2] K. Czajkowski, S. Fitzgerald, I. Foster and C. Kesselman, "Grid information Services for Distributed Resource Sharing", Proceedings $10^{\text {th }}$ IEEE International Symposium on High Performance Distributed Computing, (2002), pp. 181-184.

[3] P. May, H. C. Ehrlich and T. Steinke, "ZIB Structure Prediction Pipeline: Composing a Complex Biological Workflow through Web Services", In: Nagel, W.E., Walter, W.V., Lehner, W. (eds.) EuroPar 2006. LNCS, Springer, Heidelberg, vol. 4128, (2006), pp. 1148-1158.

[4] I. Foster and C. Kesselman, "The Grid: Blueprint for a New Computing Infrastructure", Morgan Kaufmann, San Francisco, (1999).

[5] I. Foster, C. Kesselman, J. Nick and S. Tuecke, "The Physiology of the Grid: an Open Grid Services Architecture for Distributed Systems Integration”, Technical report, Global Grid Forum, (2002).

[6] H. Hwan Kim, "Study on the Implementation of the Three-Dimensional Position Tracking Algorithm in the Indoor Environments Soonchunhyang University, (2014).

\section{Authors}

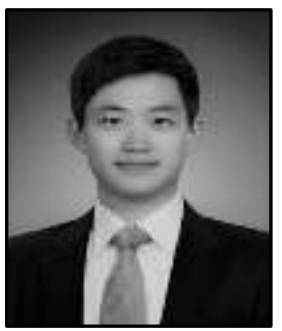

In-Kyum Kim, received the B.S degree from Hanseo University, Korea in 2017.

Author's research interests include avionics, embedded system, Indoor Flight, UAV

Currently, as a student on M.S. course at Hanseo University, Korea.

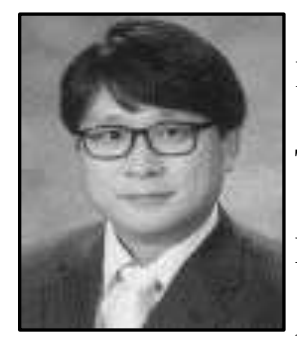

Won-Hyuck Choi, received the $\mathrm{Ph}$. D. degree in avionics from Korea Aerospace University, Korea.

Author have been a professor of smart network at Doowon Technical University College, Korea.

Currently, as a professor on Dept. of Avionics Engineering at Hanseo University, Korea since 2014.

Present research interests include embedded system, home network

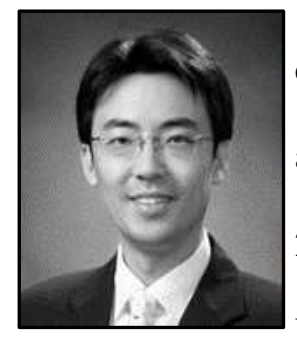

Min-Seok Jie (Corresponding Author), received the $\mathrm{Ph}$. D. degree in avionics from Korea Aerospace University, Korea.

Author was a Researcher of Intelligent Robot Reseach Center at Korea Institute of Science and Technology from 2007 to 2008.

And, as a visiting professor at Purdue University, US from 2015 to 2016.

Currently, as a professor on Dept. of Avionics Engineering at Hanseo University, Korea since 2008.

Present research interests include robust control, robot, vision based control and UAV. 